\title{
Múltiples cursos de corticoides antenatales para el parto prematuro (MACS): un estudio randomizado controlado (1)
}

\begin{abstract}
Murphy KE, Hannah ME, Willan AR, Hewson SA, Ohlsson A, Kelly EN, Matthews SG, Saigal S, Asztalos E, Ross S, Delisle MF, Amankwah K, Guselle P, Gafni A, Lee SK, Armson BA; MACS Collaborative Group. Multiple courses of antenatal corticosteroids for preterm birth (MACS): a randomised controlled trial. Lancet 2008;372(9656):2143-51.
\end{abstract}

Análisis crítico: Claudio Vera P-G. ${ }^{1,2}$, Jorge Carvajal C., PhD. ${ }^{1}$

${ }^{1}$ Unidad de Medicina Materno-Fetal, Departamento de Obstetricia y Ginecología, ${ }^{2}$ Unidad de Medicina Basada en Evidencia, Facultad de Medicina, Pontificia Universidad Católica de Chile.

\section{RESUMEN (1)}

Antecedentes: Un curso de corticoides antenatales reduce el riesgo de síndrome de dificultad respiratoria y muerte neonatal. Dosis semanales administradas a una mujer que permanece sin parir luego de un curso único, puede tener beneficios (menos morbilidad respiratoria) o daño (crecimiento fetal reducido). Objetivo: Determinar si múltiples cursos de corticoides reducen la morbilidad y mortalidad, sin afectar de modo adverso el crecimiento fetal. Métodos: 1858 mujeres entre 25-32 semanas de gestación, quienes no parieron luego de 14-21 días desde un curso inicial de corticoides antenatales y continuaban en alto riesgo de parto prematuro, fueron asignadas al azar a recibir múltiples cursos antenatales de corticoides $(n=937)$ o placebo $(n=921)$, cada 14 días hasta las 33 semanas o el parto. El resultado primario fue un compuesto de mortalidad perinatal o neonatal, síndrome de dificultad respiratorio severo, hemorragia intraventricular (grado III o IV), leucomalacia periventricular, displasia broncopulmonar o enterocolitis necrotizante. Análisis fue por intención de tratar. Todos los pacientes y encargados del cuidado desconocían el tratamiento dado. El estudio fue registrado con el número ISRCTN2654148. Resultados: Los infantes expuestos a múltiples cursos antenatales de corticoides tienen similar morbilidad y mortalidad que aquellos expuestos a placebo (150 [12,96\%] vs $143[12,5 \%])$. Aquellos que reciben múltiples dosis de corticoides pesaron menos al nacimiento que aquellos expuestos a placebo ( $2216 \mathrm{~g}$ vs $2330 \mathrm{~g}, \mathrm{p}=0,0026)$, fueron de menor talla $(44,5 \mathrm{~cm}$ vs $45,4 \mathrm{~cm}, p<0,001)$ y tenían una circunferencia craneana menor $(31,1 \mathrm{~cm}$ vs $31,7 \mathrm{~cm}, p<0,001)$. Conclusión: Múltiples cursos de corticoides antenatales, cada 14 días, no mejoran el resultado del parto prematuro, y se asocian con menor peso, talla y circunferencia craneana al nacer. Por lo tanto, este esquema de tratamiento no es recomendable.

\section{ANÁLISIS DE LA INVESTIGACIÓN}

\section{A.Relevancia clínica de la investigación}

En embarazadas en riesgo de parto prematuro que han recibido un curso completo de corticoides antenatales para inducción de madurez pulmonar: ¿la administración repetida de cursos de corticoides mejora el pronóstico perinatal?

Escenario clínico: Los recién nacidos prematuros se exponen a un elevado riesgo de morbilidad y mortalidad perinatal. Se ha demostrado que la administración de corticoides a la embarazada de betametasona $12 \mathrm{mg}$, im, cada 24 horas por dos 
veces, al menos 48 horas antes del parto, reduce la morbilidad y mortalidad perinatal, sin efectos adversos conocidos para la madre y/o el feto (2). Se ha demostrado que este efecto benéfico de los corticoides se mantiene por 7 días desde su administración (3). Por este motivo se sugirió como una buena práctica clínica, la repetición semanal de cursos de corticoides a las mujeres que luego de un primer curso de corticoides, permanecen en riesgo de parto prematuro. Las investigaciones de diseño retrospectivo pusieron en duda la seguridad y eficacia de esta práctica $(4,5)$, y los estudios prospectivos randomizados hasta ahora publicados no permiten una conclusión definitiva (6). Es indispensable contar con estudios de diseño prospectivo que permitan responder si el uso de dosis repetidas de corticoides en mujeres en riesgo de parto prematuro, resultan en más beneficio que daño, o viceversa.

\section{B.El estudio (1)}

Diseño: Estudio clínico controlado randomizado en 80 centros repartidos en 20 países. Pacientes: 1858 embarazadas (con 2318 fetos) entre 25 y 32 semanas, sin parto luego de 14 a 21 días después de la dosis inicial de corticoesteroides y que se mantenían con riesgo de parto prematuro. No fueron elegibles como participantes las embarazadas con contraindicación al uso de corticoesteroides, con uso crónico de corticoesteroides, con fetos por- tadores de anomalías congénitas letales, cursos de corticoesteroides antes de las 23 semanas, o que hubiesen participado en embarazo anterior en el estudio. Intervención: 937 embarazadas (1171 fetos) recibieron corticoides antenatales en dosis repetidas de cursos de betametasona (12 $\mathrm{mg} I \mathrm{IM}$ de 2 dosis separadas por 24 horas), cada 2 semanas, hasta las 33 semanas o el parto. Se compararon con 921 embarazadas (1147 fetos) que recibieron placebo IM de 2 dosis separadas por 24 horas, cada 2 semanas hasta el parto o las 33 semanas de edad gestacional. Resultados: No se observaron diferencias en ninguno de los componentes individuales ni en los otros resultados evaluados, incluyendo complicaciones infeccionas neonatales y maternas. No se observaron diferencias en los subgrupos de neonatos menores de 32 semanas, así tampoco en el subgrupo de nacidos antes de 7 días de la última dosis de corticoides.

El outcome compuesto principal, definido como mortalidad perinatal o neonatal, o morbilidad neonatal; la mortalidad perinatal fue definida como óbitos o muertes neonatales ocurridas durante los primeros 28 días de vida o antes del alta hospitalaria; la morbilidad neonatal significativa incluyó el síndrome de distrés respiratorio severo, displasia broncopulmonar, hemorragia intraventricular grados III o IV, leucomalacia periventricular y enterocolitis necrotizante.

\begin{tabular}{|c|c|c|c|c|}
\hline Outcome & $\begin{array}{l}\text { Dosis } \\
\text { repetidas* }\end{array}$ & $\begin{array}{l}\text { Dosis } \\
\text { única* }^{*}\end{array}$ & $\begin{array}{l}\text { Diferencia media } \\
\quad \text { (IC 95\%) }\end{array}$ & valor $p$ \\
\hline Compuesto & $150 / 1164$ & $143 / 1140$ & 1,04 & 0,83 \\
\hline principal & $(13 \%)$ & $(13 \%)$ & $(0,77$ a 1,39$)$ & \\
\hline \multirow[t]{2}{*}{ DR severo } & $87 / 1121$ & $77 / 1100$ & 1,14 & 0,82 \\
\hline & (8\%) & (7\%) & $(0,80$ a 1,58$)$ & \\
\hline \multirow[t]{2}{*}{ Peso al nacer (g) } & 2216 & 2330 & $-113,1$ & 0,003 \\
\hline & $( \pm 28,3)$ & $( \pm 28,7)$ & $(-187,0$ a $-41,17)$ & \\
\hline CC media & 31,1 & 31,7 & $-0,6$ & $<0,001$ \\
\hline (cm) & $( \pm 0,1)$ & $( \pm 0,1)$ & $(-0,90$ a $-0,32)$ & \\
\hline Talla al nacer & 44,5 & 45,4 & $-0,9$ & $<0,001$ \\
\hline (cm) & $( \pm 0,2)$ & $( \pm 0,4)$ & $(-1,34$ a $-0,37)$ & \\
\hline
\end{tabular}

* Resultados expresados como $\mathrm{n}(\%)$ o promedio ( \pm error estándar). DR: distrés respiratorio. CC: circunferencia cranearia. 


\begin{tabular}{lcccc}
\hline $\begin{array}{l}\text { Outcome compuesto } \\
\text { principal }\end{array}$ & $\begin{array}{c}\text { Dosis } \\
\text { repetidas }\end{array}$ & $\begin{array}{c}\text { Dosis } \\
\text { única }\end{array}$ & $\begin{array}{c}\text { RRR } \\
(\text { IC } 95 \%)\end{array}$ & $\begin{array}{c}\text { RAR } \\
(\text { IC } 95 \%)\end{array}$ \\
\hline En embarazos & $88 / 737$ & $83 / 726$ & $-5 \%$ & $-0,006$ \\
únicos & $(11 \%)$ & $(12 \%)$ & $(-34 \%$ a $24 \%)$ & $(0,039$ a 0,027)
\end{tabular}

RRR: Reducción de riesgo relativo, calculado con los datos reportados con CATMAKER®.

RAR: Reducción absoluta de riesgo. Con los datos reportados sólo fue posible calcular los estimadores para embarazos únicos dado que para embarazos múltiples se requiere ajustar con los datos originales.

\section{C.Análisis crítico}

Validez interna: Estudio con bajo riesgo de sesgo que describe medidas de seguridad implementadas en diversas fases del estudio: criterios de inclusión, randomización, ocultamiento de la secuencia, ciego, seguimiento y análisis. Resultados: Outcome compuesto principal orientado al resultado perinatal incluye componentes relevantes en torno a la patología respiratoria del neonato y morbilidad asociada a prematurez. Si bien los componentes no son de la misma importancia clínica, y la frecuencia de los componentes es dominada por el resultado de distrés respiratorio severo, los resultados muestran en forma consistente ausencia de diferencia significativa para la intervención en cada uno de los componentes evaluados. El análisis de subgrupos en menores de 32 semanas, o nacidos con latencia de menos de 7 días, son consistentes con los resultados globales por lo cual no se visualizan subgrupos en los cuales exista beneficio potencial del uso de dosis repetidas. Destaca también el compromiso de talla, circunferencia craneana y peso al nacimiento, relacionados al uso de dosis repetidas. Su traducción clínica en la actualidad es desconocida y el seguimiento a largo plazo de estos niños ayudará a un mejor entendimiento de los efectos de los corticoesteroides antenatales. Comentarios: Estudio randomizado de mayor poder estadístico existente, que no muestra beneficios de las dosis repetidas de corticoesteroides antenatales cada 2 semanas sobre la mortalidad y morbilidad neonatal, comparado con dosis única. Efectos encontrados sobre la talla, circunferencia craneana y peso fetal deberán ser
Randomizado:

Ocultamiento de la secuencia de randomización:

Grupos similares al inicio:

Ciego:

- Pacientes

- Tratantes

- Recolectores de datos

- Adjudicador de eventos

- Analistas o investigadores

Análisis por intención de tratar:

Seguimiento:
SI

SI, randomización centralizada

SI

SI

SI

SI

SI

No especificado

SI

El riesgo de sesgo por falla de seguimiento se estima mínimo: no se consideró en el análisis el resultado perinatal de 12 fetos, 5 pérdidas de seguimiento y 9 exclusiones (óbitos en múltiples previos a randomización) $12 / 2318$ (5\%). 
estudiados a largo plazo para conocer su impacto sobre resultados clínicos relevantes. La actualización de la revisión sistemática previa, proveerá información más detallada de los efectos de dosis repetidas (6). Conclusión: No se recomienda el uso de dosis repetidas de corticoides para las pacientes en riesgo de parto prematuro.

\section{BIBLIOGRAFÍA}

1. Murphy KE, Hannah ME, Willan AR, Hewson SA, Ohlsson A, Kelly EN, Matthews SG, Saigal S, Asztalos E, Ross S, Delisle MF, Amankwah K, Guselle P, Gafni A, Lee SK,Armson BA; MACS Collaborative Group. Multiple courses of antenatal corticosteroids for preterm birth (MACS): a randomised controlled trial. Lancet 2008;372(9656):2143-51.

2. Roberts D, Dalziel S. Antenatal corticosteroids for accelerating fetal lung maturation for women at risk of preterm birth. Cochrane Database Syst Rev. 2006; 3: CD004454.
3. NIH. Effect of corticosteroids for fetal maturation on perinatal outcomes. NIH Consens Statement 1994;12:124.

4. Newnham JP, Moss TJ. Antenatal glucocorticoids and growth: single versus multiple doses in animal and human studies. Semin Neonatol 2001;6:285-92.

5. Wapner RJ, Sorokin Y, Mele L, Johnson F, Dudley DJ, Spong CY, et al. Long-term outcomes after repeat doses of antenatal corticosteroids. N Engl J Med 2007;357:1190-8.

6. Crowther CA, Harding JE. Repeat doses of prenatal corticosteroids for women at risk of preterm birth for preventing neonatal respiratory disease. Cochrane Database Syst Rev. 2007: CD003935.

Declaración de conflicto de interés: El autor Claudio Vera participó como colaborador del estudio MACS en Chile, siendo el investigador responsable en la Pontificia Universidad Católica de Chile. 